\title{
Dibujar, especular y más dibujar. Los Carducho y Cajés, entre la formación italiana y la escuela española
}

\author{
Álvaro Pascual Chenel \\ Universidad Complutense de Madrid \\ alpchenel@ucm.es
}

RESUMEN: Vicente Carducho y Eugenio Cajés fueron dos de los pintores españoles de formación italiana más importantes en el ámbito cortesano de la primera mitad del siglo XVII. Ambos fueron, además, figuras clave en la conformación de la escuela madrileña y en su prolongación en la segunda mitad de siglo. No solo colaboraron juntos en multitud de encargos, sino que en sus respectivos obradores se formaron buena parte de los pintores que destacarían en la segunda mitad del siglo XVII.

El presente artículo se centra en la figura de ambos artistas como personajes estrechamente vinculados y se presentan dibujos y pinturas inéditas que amplían sus catálogos en ambas facetas, así como reflexiones y aproximaciones sobre la estrecha relación personal entre ambos y su obra gráfica.

PALABRAS CLAVE: Bartolomé Carducho; Vicente Carducho; Eugenio Cajés; Dibujo; Pintura; Siglo XVIl; Escuela madrileña.

\section{Dibujar, Especular y más Dibujar. The Carduchos and Cajés, between the Italian Training and the Spanish School}

ABSTRACT: Vicente Carducho and Eugenio Cajés were two of the most important Italian trained Spanish painters working in the Court of Spain of the first half of the $17^{\text {th }}$ century. Both were key figures in the creation of the Madrid school and of its extension and expansion in the second half of the century. They not only collaborated together in a multitude of commissions, but some of the outstanding painters on the second half of the $17^{\text {th }}$ century were formed in their respective studios.

The present article focuses on the figures of both artists as closely related personages. To do so this paper presents and analyses unknown drawings and paintings that expand their catalogues in both facets of their artistic production, and introduces new approaches of the close personal and professional relationship between the two artists.

KEYWORDS: Bartolomé Carducho; Vicente Carducho; Eugenio Cajés; Drawing; Paintig; $17^{\text {th }}$ Century; School of Madrid.

Recibido: 28 de febrero de 2018 / Aceptado: 7 de enero de 2019.

A mis queridos Isabel y Ángel, sin los que nada de todo esto hubiese sido posible

El dibujo es, dentro de la producción de un artista, el elemento más fresco, vivo e inmediato de su proceso creativo. Nos acerca del modo más directo e íntimo con la plasmación material y física de su primera idea y pensamiento que después elabora y reelabora hasta llegar a la obra acabada, ya sea pintura, escultura, arquitectura, etc., de tal modo que se convierte en base fundamental del aprendizaje y parte esencial en el proceso intelectual de creación artística, tal como propugnaban los teóricos desde el Renacimiento. Además, es un medio versátil de enormes posibilidades y capacidad de adaptación a las múltiples facetas artísticas y a las amplias y variadas labores pictóricas más allá de la pintura mural o de caballete: escultura, grabado, decoraciones teatrales, composiciones arquitectónicas, decoraciones efímeras, etc ${ }^{1}$.

Cómo citar este artículo: PASCUAL CHENEL, Álvaro, "Dibujar, especular y más dibujar. Los Carducho y Cajés, entre la formación italiana y la escuela española», Boletín de Arte-UMA, n. ${ }^{\circ}$ 40, Departamento de Historia del Arte, Universidad de Málaga, 2019, pp. 281-293, ISSN: 0211-8483, e-ISSN: 2695-415X, DOI: http://dx.doi.org/10.24310/ BoLArte.2019.v0i40.6496 
La primera generación, los pintores italianos en El Escorial: Patricio Cajés (h. 1540-1612) y Bartolomé Carducho (1560-1608)

Patricio Cajés y Bartolomé Carducho vinieron a trabajar a El Escorial, que, recién terminado, reclamaba abundantes labores pictóricas decorativas tanto murales como de caballete. Como colaboradores o discípulos, formaban parte del grupo de prestigiosos pintores italianos reclamados por Felipe II, en los que la tradición en la práctica del dibujo constituía el fundamento de los procesos formativos y de creación artística. Entre ellos cabe destacar a Romulo Cincinnato (h. 1540-1597), Francesco de Urbino (h. 1545-1582), Pellegrino Tibaldi (1527-1596), Giovanni Battista Castello, il Bergamasco (1509-1569) y Federico Zuccaro (h. 1542-1609). Estos artistas resultan especialmente relevantes para el futuro desarrollo de la teoría y la práctica del dibujo en España, no solo por la influencia técnica, metodológica, estilística y estética que ejercieron en sus discípulos y las generaciones de pintores formados en su círculo, sino también, en el caso específico de Zuccaro, por su fundamental labor de tratadista. De este modo, El Escorial se convertía en un centro artístico y crisol cultural que habría de tener notables consecuencias para la formación, difusión de métodos, prácticas, técnicas, formas, modelos y conceptos italianos en las generaciones subsiguientes, que los asimilaron y desarrollaron.

Patricio y Bartolomé se establecieron definitivamente en España llegando a ocupar ambos los principales oficios pictóricos al servicio de la corona. De este modo, se pueden establecer interesantes paralelismos como si de una constante se tratase que se repiten en dos generaciones sucesivas. Patricio y Bartolomé se convertirán en nexo de unión entre los artistas nacidos y educados en Italia, con la siguiente generación, a la que pertenecen sus más directos discípulos, Eugenio Cajés y Vicente Carducho, hijo y hermano de los anteriores respectivamente, nacidos -en el caso de Eugenio- y formados ya en España, pero en el ambiente claramente italianizante que suponía El Escorial, además del posible viaje de estudios a Italia de ambos, asunto que merecería una mayor profundización².

Tanto Patricio como Bartolomé pondrán en práctica lo aprendido en el ambiente formativo toscano-romano en sus trabajos en España y, tal como después repetirán su hijo y hermano, colaborarán en varias empresas conjuntas a lo lar- go de su carrera, como en el palacio de El Pardo, el retablo no concluido de San Felipe el Real, así como varios trabajos para clientes privados en Madrid (Angulo y Pérez, 1969: 14-15).

Bartolomé Carducho se trasladó de su Florencia natal a España en 1585 acompañando a su maestro Federico Zuccaro para trabajar en El Escorial. Ahora podemos añadir una pintura inédita de Bartolomé Carducho que incorporar a su corpus pictórico. Se trata de una imponente Asunción de la Virgen, firmada y fechada por el artista en $1595^{3}$, perteneciente a la colección Granados de Madrid [1]. Desde luego podría ponerse en conexión con el dibujo atribuido a Vicente Carducho de la Real Academia de Bellas Artes de San Fernando 4 (Angulo y Pérez, 1977: 156, n. 523) que, en realidad, está más relacionado con el propio Bartolomé o, en todo caso con algún discípulo de Vicente como Félix Castello; así como con el fresco de idéntico asunto que pintara Vicente en la capilla del Sagrario de la catedral de Toledo (Angulo y Pérez, 1969: 186-187). Como resulta lógico, Vicente seguía los modelos de su hermano y maestro, algo que será constante en numerosas ocasiones a lo largo de su vida.

\section{La formación italiana en España. Vicente Carducho} (h. 1576/1578-1638) y Eugenio Cajés (1574-1634)

En efecto, Bartolomé trajo consigo a España a su hermano Vicente, un niño de apenas 7 o 9 años que se convertiría en su discípulo y heredaría tras su muerte en 1608 el título de pintor del rey. En los últimos años se ha venido revalorizando cada vez más la importancia de su figura, ponderando su trascendencia en el peculiar escenario cortesano y situándolo en el lugar histórico-artístico que le corresponde en una época en la que la alargada sombra de la genialidad y novedad de Velázquez oscureció historiográficamente a otros artistas contemporáneos (Pascual y Rodríguez, 2017: 2643-2730).

No resulta sencillo compendiar la complejidad y amplitud de la intensa actividad desplegada por Vicente Carducho durante su vida, así como su impacto e influencia ejercida en los artistas de su entorno. A lo largo de su dilata y prolífica carrera reunió en su persona una serie de facetas estrechamente ligadas entre sí, y recibió una gran cantidad de encargos -tanto por parte de la Corona, la nobleza, como de 
diferentes instituciones y órdenes religiosas- entre los que se encuentra uno de los más importantes de todo el siglo de Oro: el gran ciclo cartujano de El Paular. Todo ello le convirtió en uno de los pintores más prestigiosos y respetados del panorama artístico español del primer tercio del siglo XVII y su labor de magisterio le sitúa como bisagra fundamental para el posterior desarrollo de la escuela madrileña de la segunda mitad de siglo ${ }^{5}$.

Se conserva un buen número de dibujos correspondientes a las diferentes etapas de su carrera a través de los que se pude ir reconstruyendo su trayectoria vital y seguir su actividad a lo largo del tiempo. Desde sus primeros trabajos en El Escorial y Valladolid bajo la tutela de Bartolomé, pasando por las decoraciones del Pardo, hasta los numerosos trabajos en el ámbito de la corte.

Tras los trabajos en Valladolid y el Pardo, el grueso de la actividad de Vicente se centró en Madrid y sus alrededores. Desde entonces sería una constante en su vida la abundancia de trabajo, simultaneando en numerosas ocasiones varios importantes y amplios encargos. Ello determina tres consecuencias lógicas: un creciente prestigio en el ámbito palatino-cortesano; una holgada situación económica; y la necesaria articulación de un amplio obrador altamente cualificado, eficiente y perfectamente organizado en torno al maestro.

A lo largo de todos esos años que median aproximadamente entre 1613-1638, compaginó sus trabajos palatinos al servicio estable de la corona como pintor del rey bajo Felipe III y Felipe IV con otros muchos en el entorno cortesano para distintas instituciones religiosas, de modo que es raro el templo, monasterio o convento en Madrid que no contase con alguna pintura suya. Entre 1613-1621 se pueden citar los numerosos encargos para el monasterio de la Encarnación, Santo Domingo el Real, San Gil el Real (Pascual, 2017: 433-444), la Catedral de Toledo, monasterio de Guadalupe o el retablo de Algete, en los que además trabajó junto a su compañero y amigo Eugenio Cajés. A la muerte de Felipe III en 1621 se encarga también a medias con Cajés de las decoraciones fingidas para el túmulo de las exequias en San Jerónimo y seguirá trabajando intensamente reinando ya Felipe IV. El periodo comprendido entre finales de la década de 1620 y su muerte en 1638 concentra los años más intensos de su carrera. Para la corona destaca la decoración del salón de nuevo del Alcázar y la del palacio del

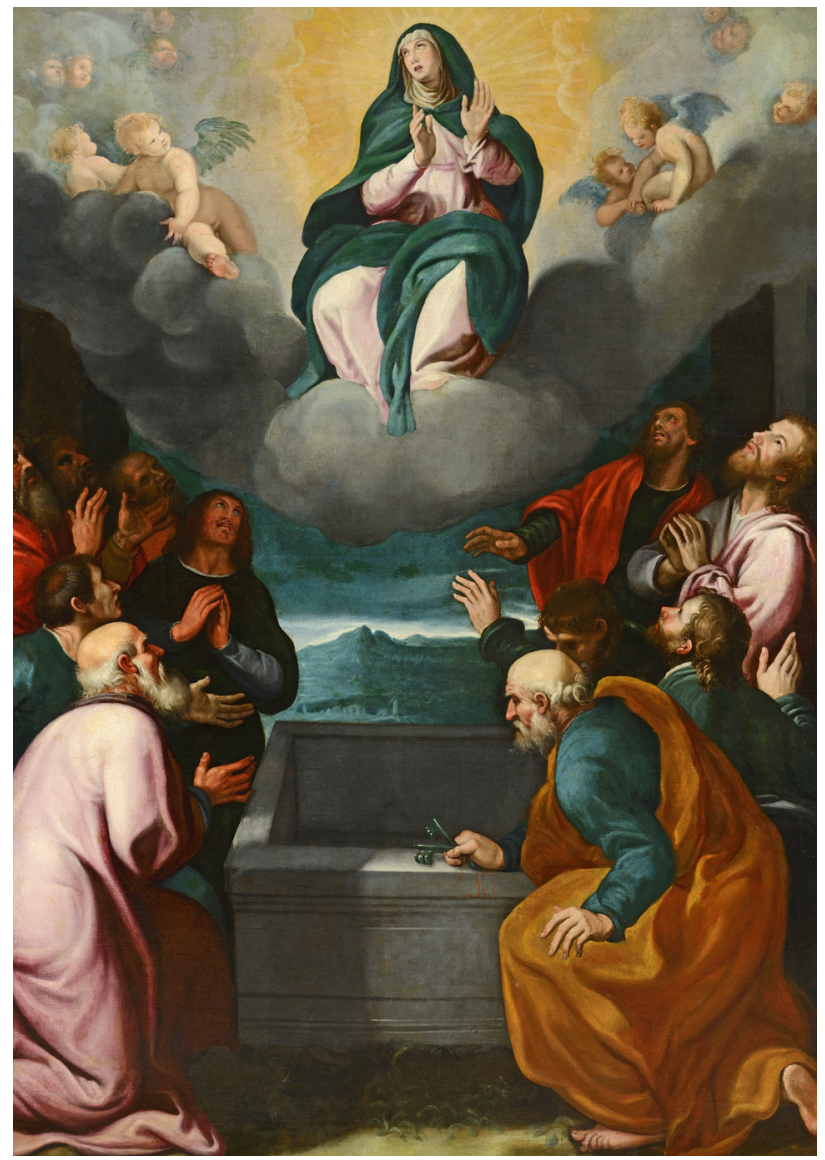

1. Bartolomé Carducho, Asunción de la Virgen, Colección Granados, Madrid

Buen Retiro. En este ámbito se enmarcan también los encargos para iglesias y conventos de patronato regio como el conjunto de los retablos de la iglesia de San Gil el Real, y aquellas obras directamente comisionadas por la clientela eclesiástica como el retablo de San Antonio de Padua para San Antonio de los Portugueses y la serie para el convento de los Trinitarios. Todo ello además se desarrolla de forma contemporánea a la que habría de ser la empresa pictórica de su vida y encargo sin parangón en la pintura española del siglo XVII: la serie de 56 cuadros destinados a la Cartuja de El Paular que le ocuparía desde 1626 a 1632, y para la que se conserva el conjunto más amplio de dibujos preparatorios del artista.

Desde la publicación del catálogo razonado de dibujos de Vicente Carducho en 2015, se han podido identificar nuevas obras gráficas del pintor. Referidas precisamente 


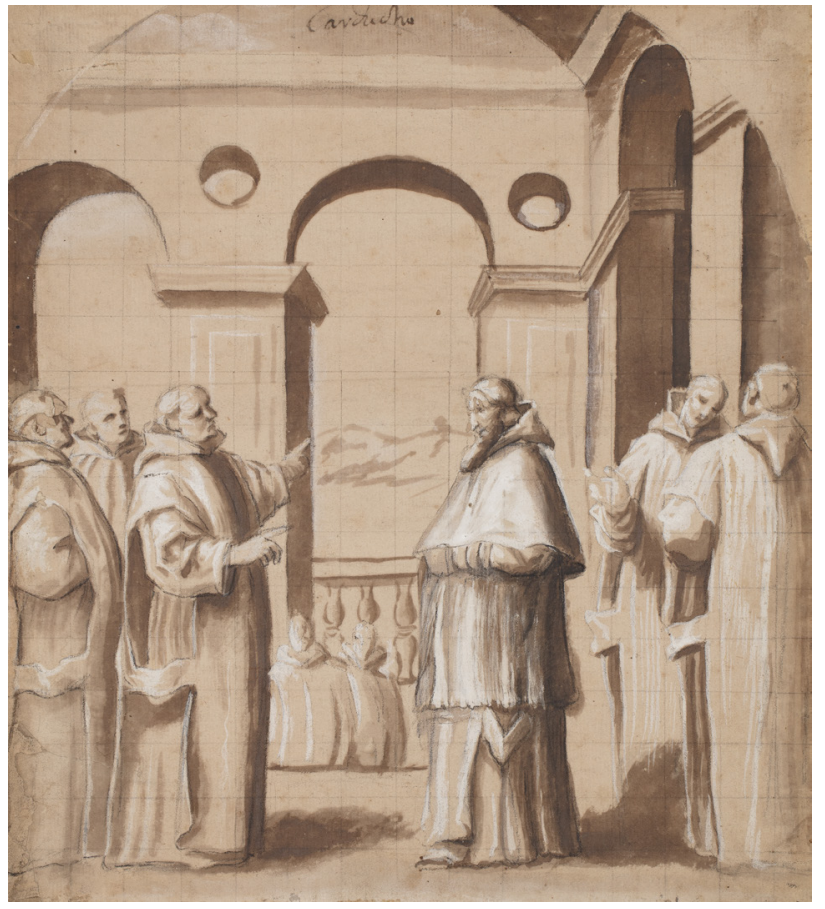

2. Vicente Carducho, San Bruno despide a San Hugo, Colección Colomer, Madrid

al ciclo de El Paular merece la pena mencionar, por su novedad y relevancia, algunas de las últimas incorporaciones que, además de su calidad, amplían aún más el corpus de la serie. Se trata de otros tres ejemplares más pertenecientes a este importante ciclo, todos ellos referidos a composiciones completas y preparatorios para sus respectivos lienzos homónimos. Uno es la Aparición de los ángeles músicos a San Hugo de Lincoln de los Uffizi ${ }^{6}$ (Navarrete, 2016: 189-190, n. 82; Pascual, 2018: 224-231). El segundo, recientemente incorporado a la colección Colomer, figura el episodio en el que San Bruno despide a San Hugo y presenta las habituales pequeñas variaciones y diferencias entre el dibujo y el cuadro definitivo (Rodríguez, en Spanish Master Drawings, 2019: 10-13) [2]. El último se conserva en el Kupferstich-Kabinett de Dresde y representa a El prior Bosón resucitando a un albañil (García-Toraño, Mezte, Pascual and Rodríguez, 2019: 197-204). Presenta una particularidad única en los dibujos hasta ahora conocidos del ciclo. Está cuadriculado para transferir como el resto; pero en este caso, el artista ha empleado distintos tamaños de cuadrícula que varían en función del nivel de detalle y dificultad para trasponer al lien- zo determinadas figuras. Esto se evidencia claramente en el personaje del albañil caído, cuya cuadrícula es mucho más pequeña respecto de la del resto de la composición. Esto añade otro elemento de estudio para profundizar en el proceso creativo del ciclo y en los métodos y procedimientos de trabajo del artista y su obrador?

Además de algunas otras novedades carduchescas ya reseñadas en la bibliografía específica (Pascual, 2018: 224231; García-Toraño, Mezte, Pascual and Rodríguez, 2019: 197-204), podríamos mencionar aquí otros dibujos relacionables con el artista o su entorno. En el mismo museo de Dresde se conservan dos figuras que podríamos considerar a medio camino entre las series de los Reyes Heráldicos y los Padres de la Iglesia. Uno de ellos representa un rey con corona cetro y un libro en la mano y el otro, de mayor calidad, a San Luis de Tolosa. Quizá no habría que descartar la posibilidad de relacionar este último con Francisco Rizi, principal discípulo de Carducho. En la Biblioteca Nacional de España se conserva un San Pedro de Alcántara que presenta asimismo los estilemas característicos de Vicente Carducho ${ }^{8}$. Como curiosidad cabe señalar que el dibujo Agamenón pidiendo a Aquiles que le devuelva a su esclava Briseida, perteneciente al ciclo decorativo del palacio de El Pardo, está realizado en un papel reaprovechado por el artista -cosa muy habitual en los obradores de la época- en cuyo verso hay un fragmento de una Anunciación atribuible a Francisco Pacheco.

Junto a Vicente Carducho, Eugenio Cajés (1574-1634) es otro de los pintores más destacados del ámbito madrileño del primer tercio del siglo XVII. Su trayectoria vital, formativa y profesional corre pareja a la de su amigo y asiduo colaborador Vicente Carducho, de modo que ambos vienen a ser una prolongación aumentada de lo que habían vivido junto a su padre y hermano respectivamente ${ }^{9}$. Al igual que Vicente con su hermano Bartolomé, Eugenio Cajés ocupó la plaza de pintor del rey de su padre Patricio a la muerte de este en 1612. Desde los inicios de su carrera colaboraría estrechamente y en multitud de ocasiones con Vicente Carducho en diferentes empresas pictóricas conjuntas como los encargos para la catedral de Toledo, Santo Domingo el Real, San Gil el Real (Pascual, 2017, pp. 433-444), el monasterio de Guadalupe, el retablo de Algete, o las decoraciones fingidas para las exequias de Felipe III en San Jerónimo ${ }^{10}$.

Esto explica las lógicas similitudes estilísticas, metodológicas, técnicas y conceptuales que confirieron a sus dibu- 
jos, aunque cada uno de ellos imprimiéndoles su particular sello personal. Nada tiene esto de extraño en dos personas de la misma edad, educados en el mismo ambiente bajo los mismos preceptos, que trabajaron juntos en multitud de ocasiones y que, además, eran amigos. Esto justifica también el frecuente uso, intercambio o «préstamo» de modelos comunes.

Desde el punto de vista técnico, Cajés se presenta como un hábil dibujante que, al igual que Caducho, recoge lógicamente el legado de los métodos y técnicas aprendidos en El Escorial junto a su padre y el resto de pintores italianos, pasándolos después por su particular tamiz para crear un estilo gráfico muy personal en el que son notas comunes la combinación de diferentes técnicas y la intensidad de los valores plástico-expresivos. Es muy destacado en Cajés su peculiar interpretación manierista de características figuras alargadas -aún más que las de Carducho- y formas vaporosas de aspecto sinuoso y llameante. Al igual que su compañero, maneja con maestría similar lenguaje gráfico destacando la combinación de técnicas en el uso del lápiz negro, las agudas de tinta parda y el abundante empleo personalísimo de la sanguina, punto en el que se diferencia de Carducho. A través de estos recursos manejados con una gran suavidad y blandura en el modelado de las formas -su característica morbidezza- consigue dotar a sus dibujos de unas calidades de elevado efecto pictórico.

En los últimos años se han identificado algunos dibujos del artista que han de ser incorporados a un catálogo razonado que está aún por hacer. Entre ellos se encuentra una Visitación en los Uffizi que Navarrete atribuye justamente a Cajés (2016: 138-139, n. 38). Las relaciones que plantea con el lienzo de Cajés del Abrazo de San Joaquín y Santa Ana en la Puerta dorada, conservado en la Academia de Bellas Artes de San Fernando son indudables, así como con el cuadro de Carducho en el Palacio Real de Madrid. Es inevitable también evocar uno los dibujos de Vicente Carducho de la Albertina con el tema del Abrazo, que presenta unos modelos y una plástica muy similar (Pascual y Rodríguez, 2015: 300-305, n. 65.2). En relación con los dibujos y pinturas arriba mencionados damos a conocer una pintura inédita de Eugenio Cajés. Se trata de un Abrazo en la Puerta Dorada, perteneciente a la colección Granados de Madrid [3]. Resulta más que evidente la vinculación directa de este lienzo con la Visitación de los Uffizi, con los dibujos de Car-

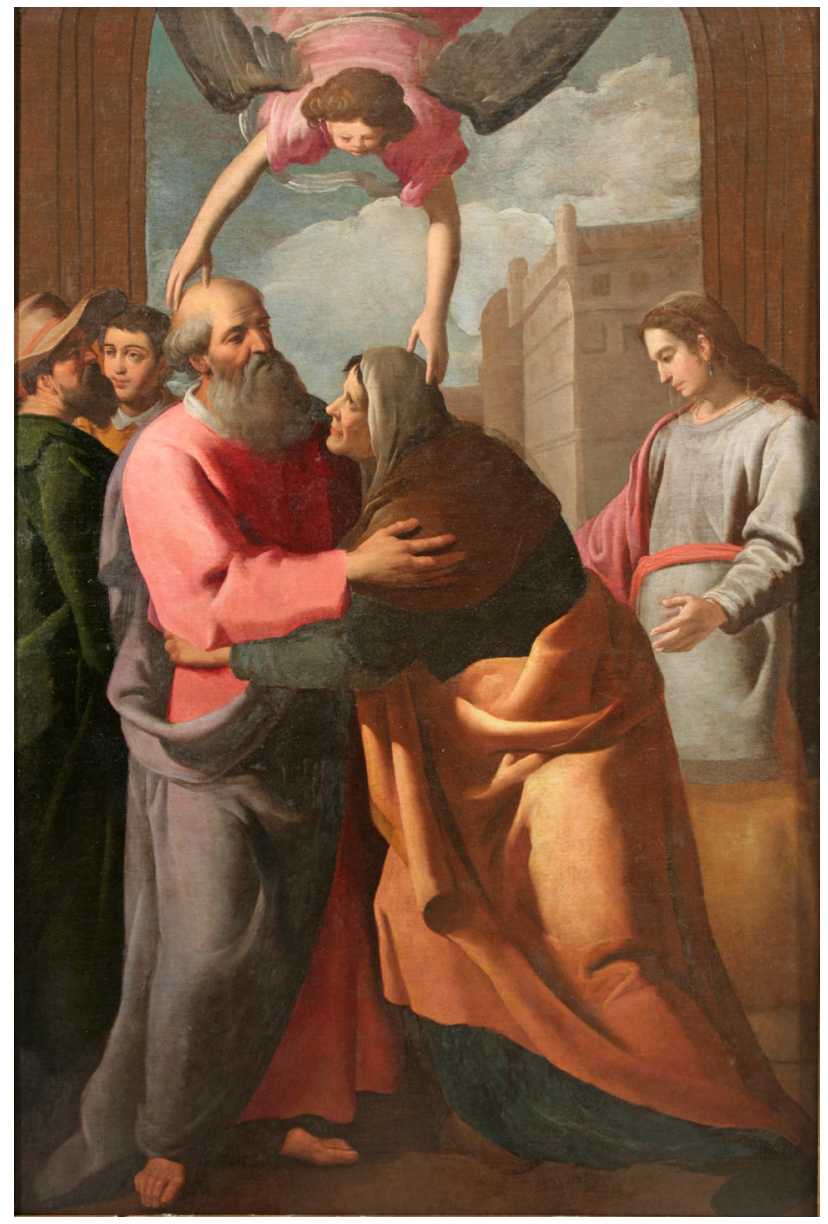

3. Eugenio Cajés, Abrazo en la Puerta Dorada, Colección Granados, Madrid

ducho de la Albertina, así como obviamente con el mencionado cuadro de la Academia de San Fernando, que formó parte del conjunto del convento de San Felipe el Real de Madrid, pintado en 1605 y donde había otras importantes obras de Cajés que perecieron en el incendio de 1718 del que esta obra se salvó. El cuadro de la colección Granados se trata de una versión más pequeña y con ligeras variantes respecto del de la Academia. Asimismo, resultan muy interesantes las correcciones o cambios que el artista hizo sobre todo en lo que se refiere a la espalda de Santa Ana y la mano izquierda del personaje femenino que la acompaña.

Como señalábamos antes, son muy frecuentes las estrechas interrelaciones e intercambio de modelos y composiciones entre Carducho y Cajés, lo que demuestra su éxito y el mantenimiento de la comunidad de intereses conjun- 


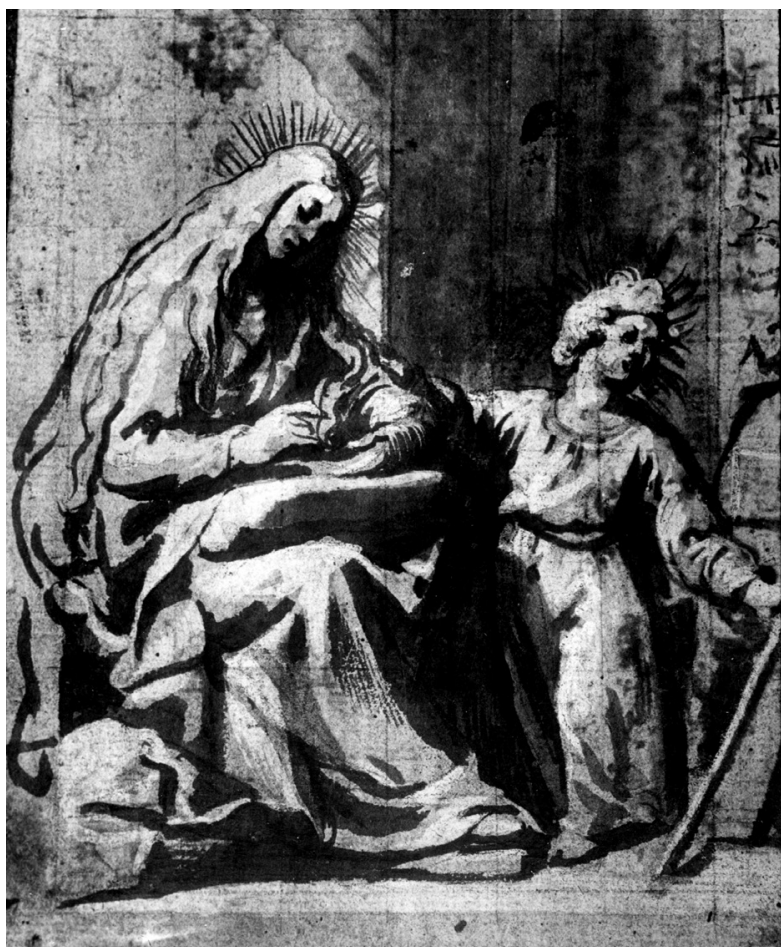

4a. Vicente Carducho (aquí atribuido), Virgen y el Niño, Museo Casa Natal de Jovellanos, Gijón

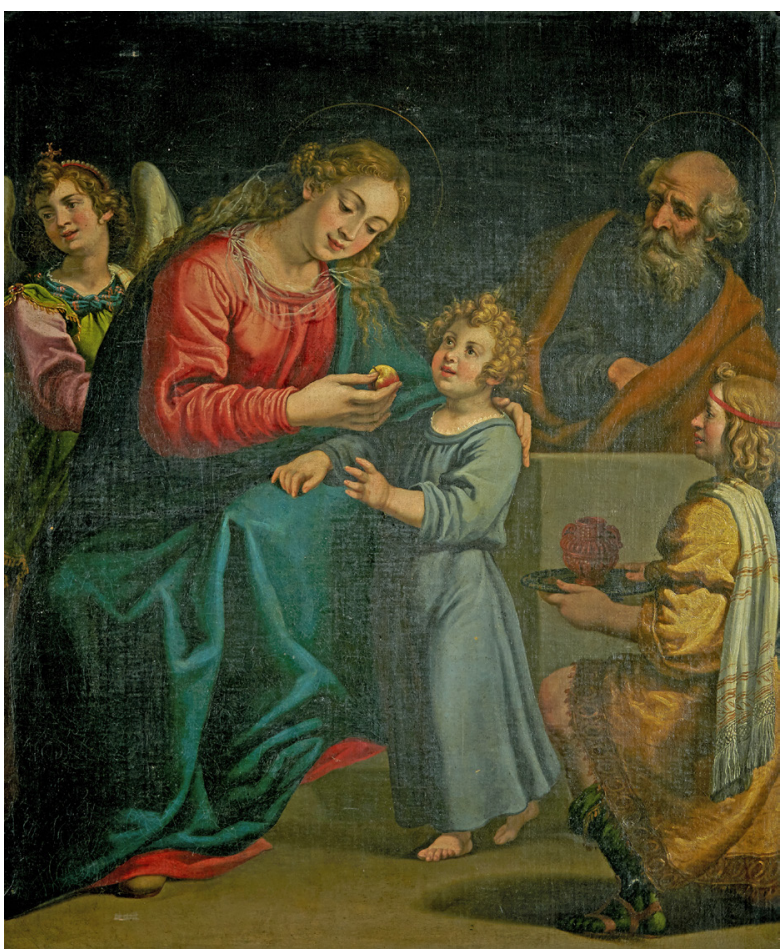

4b. Vicente Carducho, Sagrada Familia, colección particular tos, casi como si se tratase de una estrategia comercial de acaparación y monopolio pictórico ante la gran demanda de pintura religiosa. De hecho, no hubo casi ningún templo, convento o institución religiosa del Madrid de la época que no contase con alguna obra de estos artistas, lo que es síntoma claro de su prestigio y de lo demandados que estaban. Hasta el punto de que a lo largo de sus carreras compaginaron de forma simultánea varios encargos diferentes en numerosas ocasiones.

Esto resulta muy evidente por ejemplo en la compleja serie de dibujos y cuadros que se conservan de ambos artistas con el tema genérico de Cristo sentado, de los que hay ejemplares bajo diversas advocaciones como el Cristo de la Humildad y Paciencia de los Uffizi; Cristo sentado de la Biblioteca Nacional de España, o el bellísimo Cristo sentado en el Calvario de la Academia de San Fernando todos ellos de Cajés y estrechamente vinculados con varios de sus cuadros firmados. En el antiguo Instituto Jovellanos de Gijón existió un dibujo de La Virgen ante Cristo en el Calvario antes de la Crucifixión de Vicente Carducho, hoy perdido, del que se conserva su correspondiente lienzo firmado por el artista (Pascual y Rodríguez, 2015: 122-123, n. 20; Navarrete, 216: 150 , n. 48). Algo muy similar ocurre también con los diversos bocetos y pinturas de Preparativos para la flagelación de Cristo que se conocen de uno y otro. El dibujo de Cajés del Philade/phia Museum of Art se relaciona con el de Carducho en los Uffizi. Otro de Cajés hay en la Biblioteca Nacional de España ${ }^{11}$. Recientemente se ha publicado además un cuadro con esta misma iconografía, firmado por Cajés, que ha de vincularse a estos modelos, así como a su Cabeza femenina de la Real Academia de Bellas Artes de San Fernando (Pascual en La colección Delgado, 2017: 56-58).

Otras interesantes analogías pueden rastrearse a lo largo de sus respectivos catálogos. Es el caso del destruido dibujo de La Virgen y el Niño del antiguo Instituto Jovellanos, atribuido con dudas a Eugenio Cajés. Angulo y Pérez Sánchez lo recogen sin duda prudentemente entre los dibujos aceptados del artista -siguiendo las atribuciones anteriores a su pérdida- ante la imposibilidad de estudiarlo en directo. A pesar de ello y de que tenía una inscripción dieciochesca que lo asignaba a Eugenio o Patricio, su agudeza les llevó ya a plantearse serias dudas sobre la autoría, relacionándolo más bien con las características propias de Vicente Carducho, al que, en efecto, creo que debe asignarse (Angulo 
y Pérez, 1977: 15, n. 18). El único testimonio que sobrevive es una vieja fotografía que no permite juicios más afinados, pero desde luego parece de estilo próximo a La Virgen ante Cristo en el Calvario antes de la Crucifixión arriba mencionado que se encontraba en la misma institución. Más definitiva podría ser su comparación con algunos lienzos de Carducho. Parece evidente la cercanía de este Niño con el que aparece en el San Blas de la catedral de Córdoba (Pascual y Rodríguez, 2015: 166-168, n. 28). Pero sobre todo hay que mencionar la Sagrada Familia recientemente reaparecida, obra indudable del maestro (Pascual, 2017: 435), para la que creo que La Virgen y el Niño del Jovellanos podría haber sido un primer dibujo preparatorio de la composición general sobre la que, siguiendo su práctica habitual, después añadía o quitaba personajes e introducía pequeñas variantes, modificaciones o detalles en el lienzo definitivo [4].

El tema de la Virgen con el Niño en sus diversas variantes fue una iconografía muy reclamada a Cajés, que llegó a pintar numerosos ejemplares en los que subyace el sustrato común del dibujo del Museo del Prado, de factura rápida y sumaria en el que ensaya varias posiciones para la cabeza de la Virgen ${ }^{12}$; y el del Courtauld Institute de Londres, de valores pictoricistas, mucho más terminado y también cuadriculado ${ }^{13}$. En relación con dichos dibujos podemos señalar algunas pinturas inéditas o poco conocidas. Si el dibujo de la Virgen con el Niño del Courtauld puede ligarse de modo evidente con el cuadro de composición más amplia La Virgen con el Niño y ángeles del Museo del Prado ${ }^{14}$; aún ha de vincularse de modo mucho más directo con la Sagrada familia con ángeles en oración de la colección Casacuberta-Marsans, para el que es sin duda su más inmediato precedente [5]. Por su parte, el diseño del Museo del Prado ${ }^{15}$ enlaza directamente con otra Virgen con Niño, lienzo recientemente aparecido en el mercado madrileño ${ }^{16}$. De este modo, un mismo dibujo podía ser utilizado en varias ocasiones, adecuándolo para crear diferentes composiciones en las que bastaba introducir pequeñas variantes dependiendo de las necesidades del encargo.

Aún se pueden señalar algunas otras obras inéditas de Cajés que enriquecen su corpus pictórico y se vinculan además con sus dibujos. Una Trinidad en la Tierra firmada y fechada en $1616^{17}$ podría identificarse con un cuadro encargado al pintor ese año con destino a un retablo ubicado en uno de los pilares de la iglesia del convento de Mínimos de la Victoria de Madrid, donde también lo mencionan Palomino y

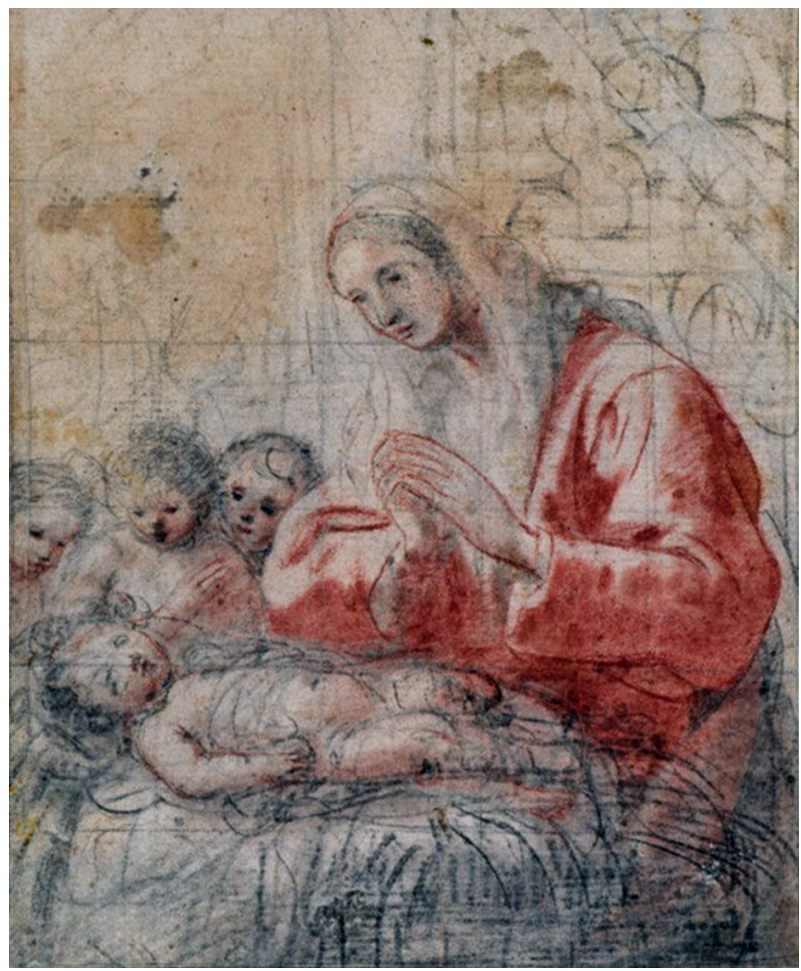

5a. Eugenio Cajés, Virgen con el Niño, The Courtauld Gallery, Londres

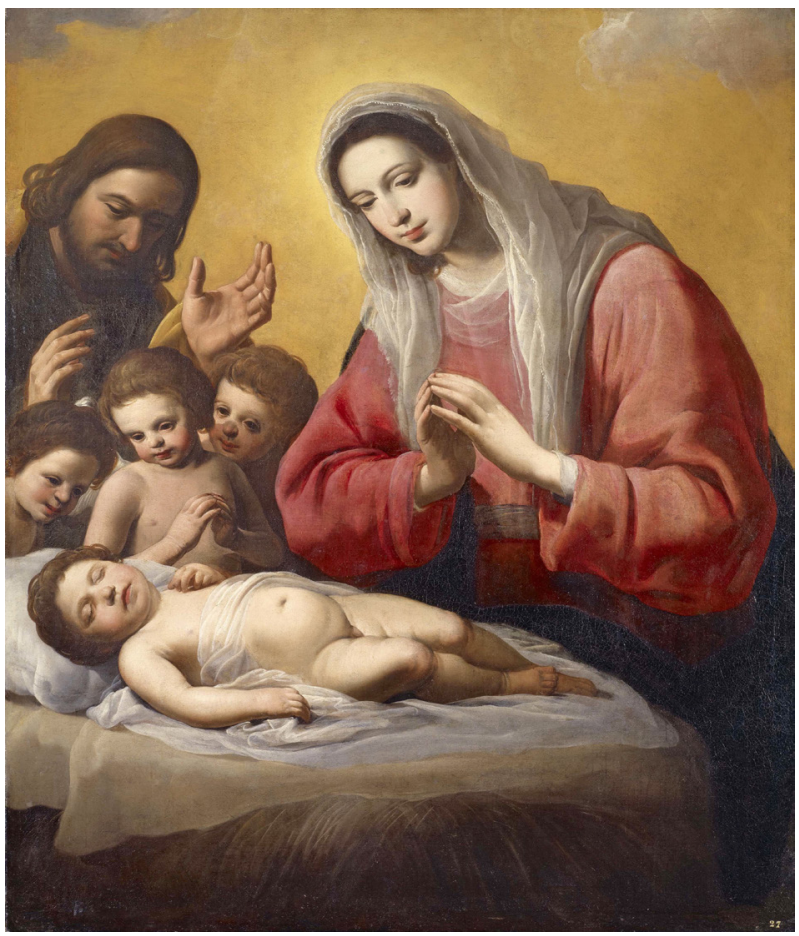

5b. Eugenio Cajés, Sagrada Familia con ángeles en oración, colección Casacuberta-Marsans 


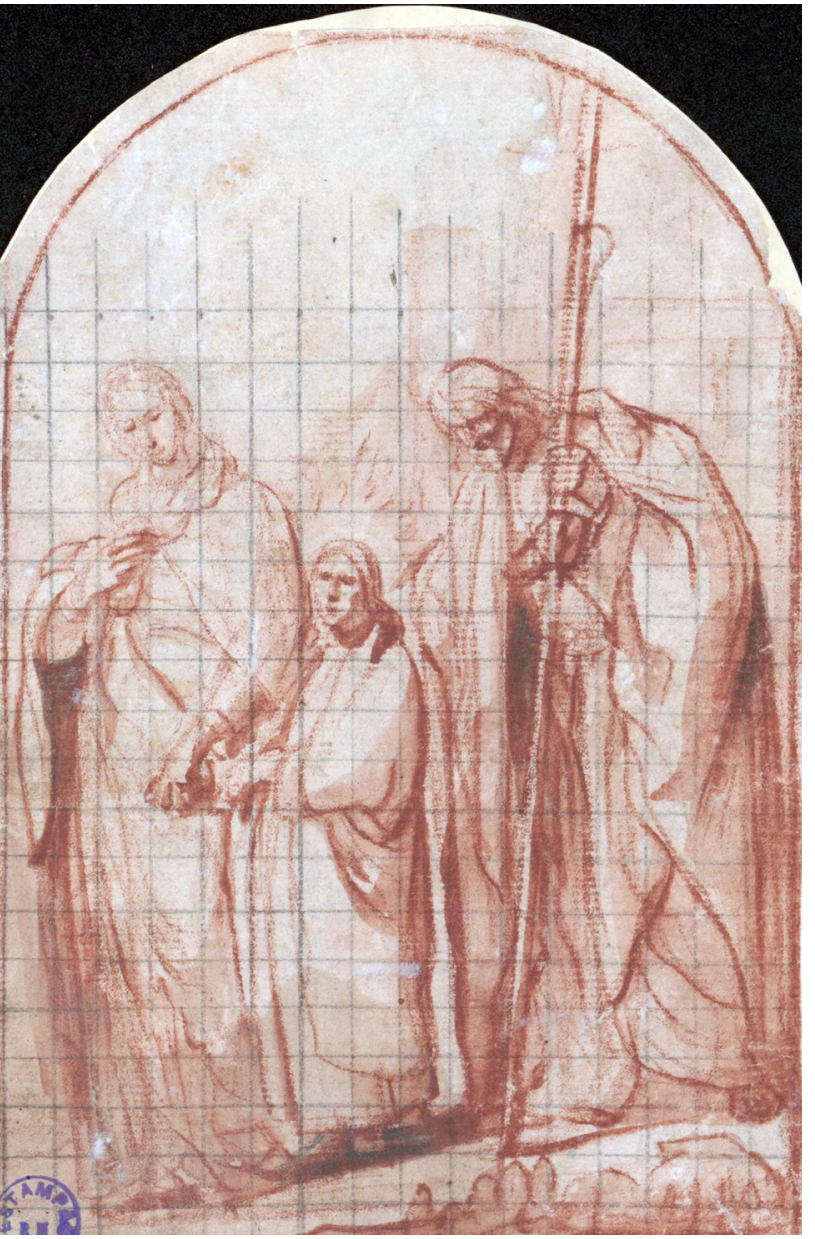

6a. Eugenio Cajés, Trinidad en la Tierra, Biblioteca Nacional de España, Madrid

Ponz ${ }^{18}$. Además de la coincidencia cronológica, su tamaño y formato concordarían con su ubicación en un retablo colocado en un pilar. De los dibujos conocidos de Cajés para este tema iconográfico los que más se ajustan son el del Museo del Prado ${ }^{19} \mathrm{y}$, sobre todo, el de la Biblioteca Nacional de España, que debe ser en efecto el boceto preparatorio (Angulo y Pérez, 1977: 16, n. 25) [6].

Notable es una Anunciación ${ }^{20}$ que parece una versión reducida de la del retablo de Algete, firmada y fechada por el artista en 1619, y con la que coincide de manera muy fiel no solo en la composición, actitudes y gestos de las figuras, sino también en los tipos humanos característicos de Cajés (Angulo y Pérez, 1969: 227, n. 1-2; Pérez, 1976: 308). El dibujo preparatorio utilizado para ambas Anunciaciones se conserva en el

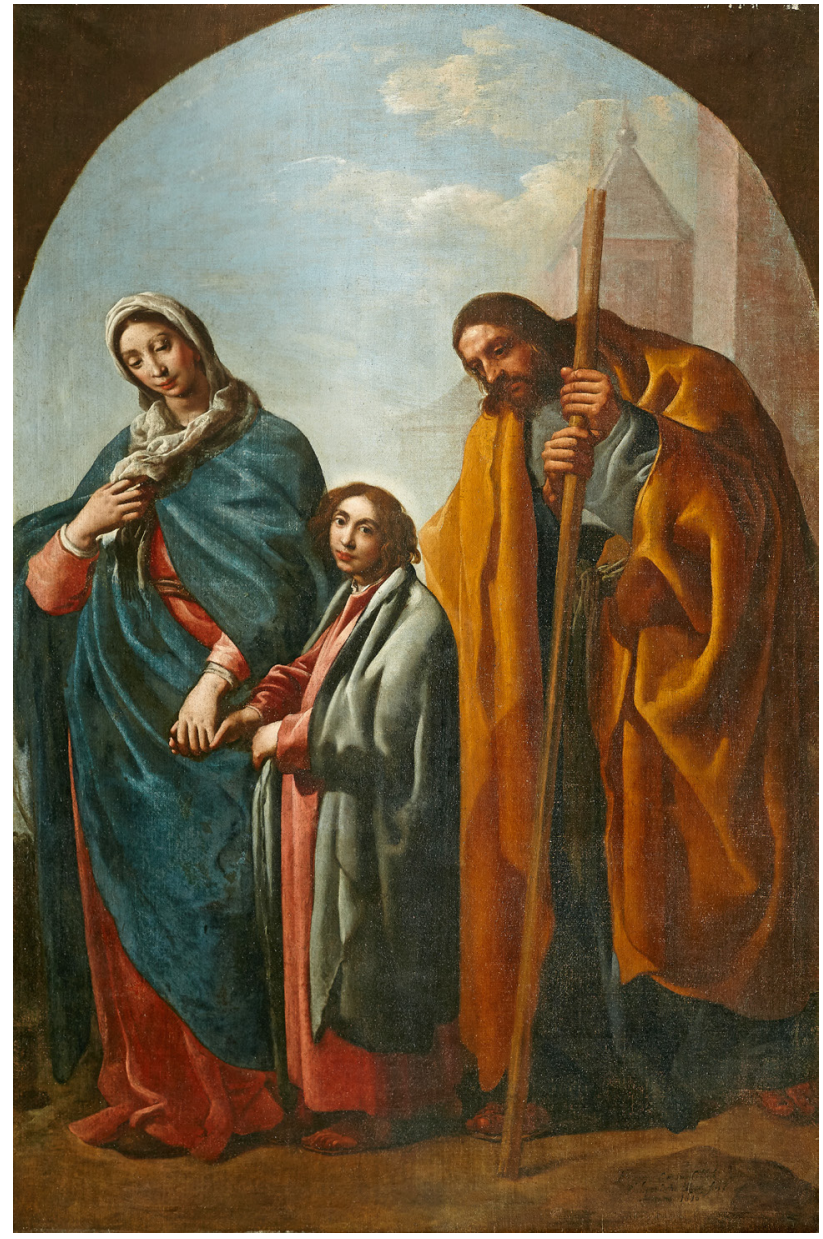

6b. Eugenio Cajés, Trinidad en la Tierra, colección particular

Museo del Prado [7], que es interesante también desde el punto de vista técnico y de los procedimientos de trabajo, pues se diferencia claramente el mayor nivel de acabado de la Virgen, así como el hecho de que la cuadrícula se centra en ella abarcando lo mínimo imprescindible de la figura del arcángel para situarlo espacialmente, procedimiento utilizado ya por los pintores italianos en El Escorial (Angulo y Pérez, 1977: 14, n. 5).

Estos ejemplos nos remiten al tema de la versatilidad del dibujo y las posibilidades de adaptación que el medio ofrecía a los artistas; al de los procedimientos de rentabilización del trabajo conservando y reutilizando prototipos como instrumentos de referencia a los que poder acudir en el futuro, etc. Obviamente Cajés empleaba los mismos métodos que Carducho. Tal como refleja el inventario de este último, 


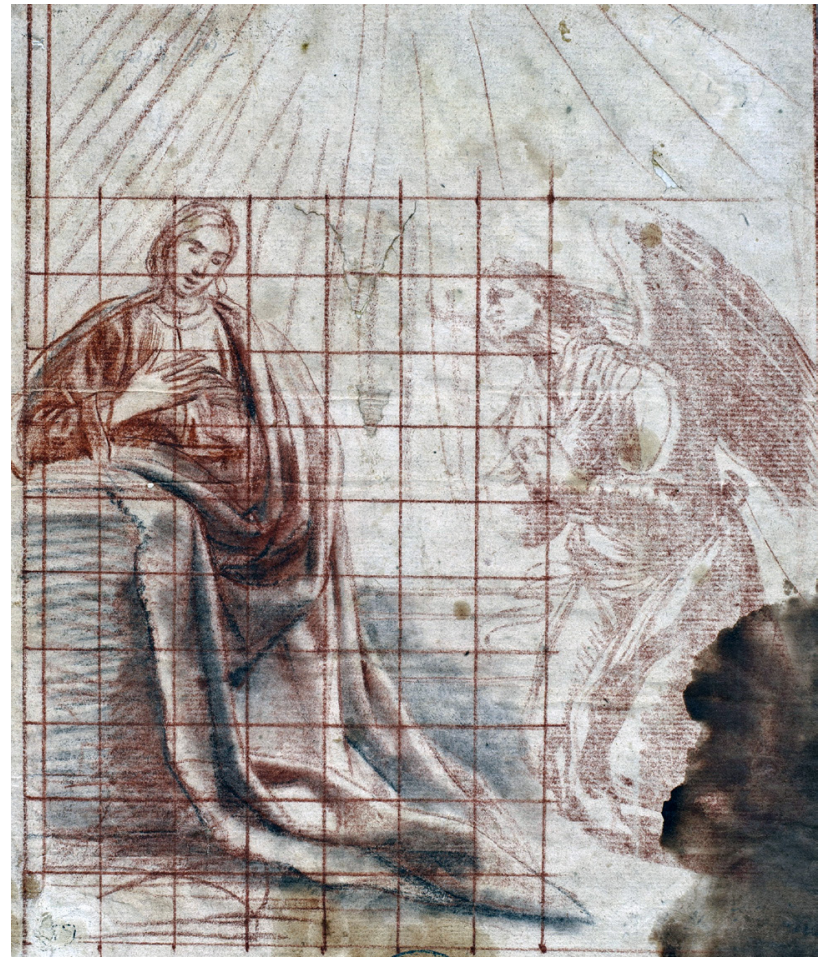

7a. Eugenio Cajés, Anunciación, Museo Nacional del Prado, Madrid

resultaba imprescindible tener siempre listo un material perfectamente organizado al que recurrir una y otra vez para componer las pinturas. Una especie de amplio catálogo o repertorio gráfico de figuras y composiciones que podían ser fácilmente adaptables según las necesidades. Es decir, se trataba de que el obrador funcionase como una verdadera factoría de producción casi en cadena con el fin de ganar eficiencia, rentabilizar el trabajo y poder atender la enorme cantidad de encargos que además se solapaban en el tiempo.

Hay que destacar otros dibujos de Cajés que añadir al catálogo de su obra gráfica. Por un lado La Dormición de la Virgen de la colección Colomer [8] ${ }^{21}$. Se trata de una obra que entronca con su estilo en los tipos humanos, el uso del lápiz y las aguadas pardas, y denota la estrecha cercanía con los dibujos de su discípulo Jusepe Leonardo. De hecho, resulta bastante próximo a algunos de los pocos dibujos conocidos de Leonardo como La Purificación de la Virgen de la Biblioteca Nacional de España; La presentación de la Virgen en el Templo de los Álbumes de Fernando VII de la Biblioteca

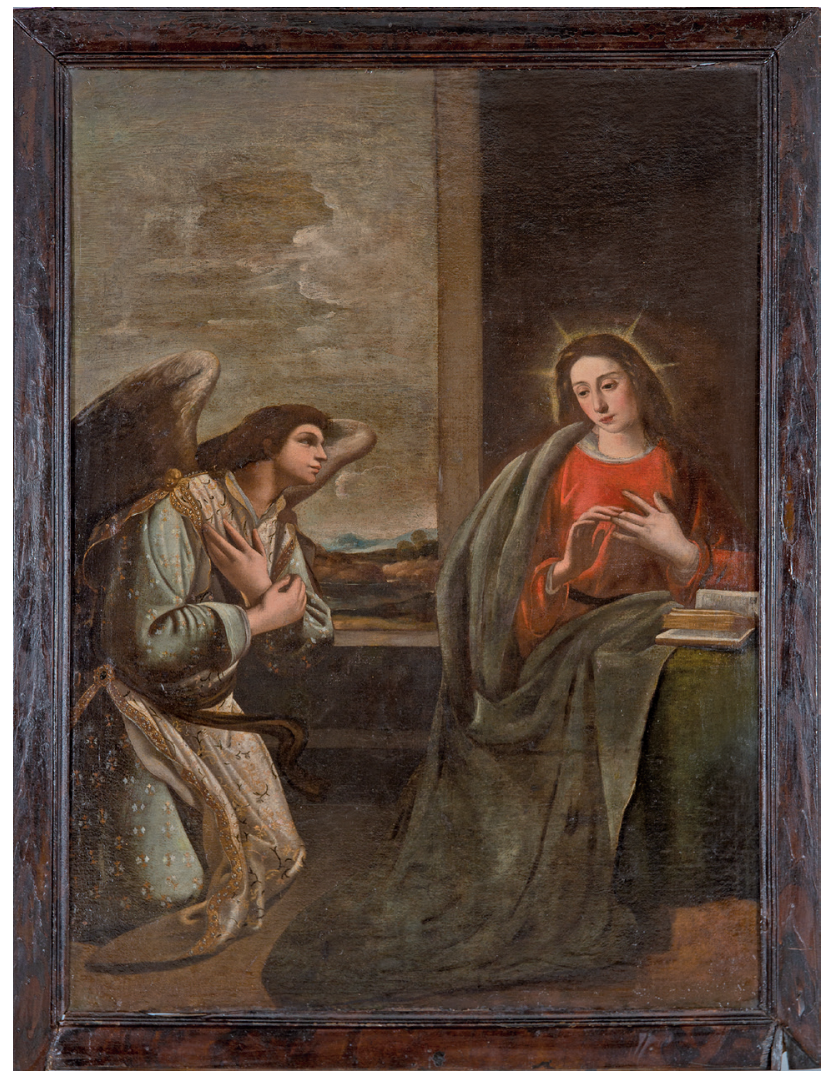

7b. Eugenio Cajés, Anunciación, colección particular

del Palacio Real de Madrid; o el Estudio de Apóstoles para la Asunción de la Virgen de los Uffizi22.

Además, añade otro ejemplar a la que debió ser importante colección de dibujos del pintor madrileño Francisco de Solís (1620-1684). A la muerte de este, su viuda encomendó a Manuel de Porras la tasación de los bienes, incluidos los dibujos, para ser vendidos en pública almoneda, de ahí la presencia de la cifra «4 Rs». Es muy probable que el propio Porras adquiriera buena parte de los dibujos de Solís, pues él mismo era coleccionista de dibujo, llegando a atesorar un importante número de ellos que además tenía en muy alta estima tal como refleja de modo claro en su testamento (Agulló, 2003: 235-248; Banner, 2007: 359-366) ${ }^{23}$. Por lo demás, la inscripción dieciochesca con la firma «Caxes», resulta muy similar a la que aparece en el dibujo de La Virgen y el Niño del antiguo Instituto Jovellanos.

Junto a él, el dibujo para un Evangelista, recientemente adquirido por Félix Palacios [9]. Se trata de una obra típica 


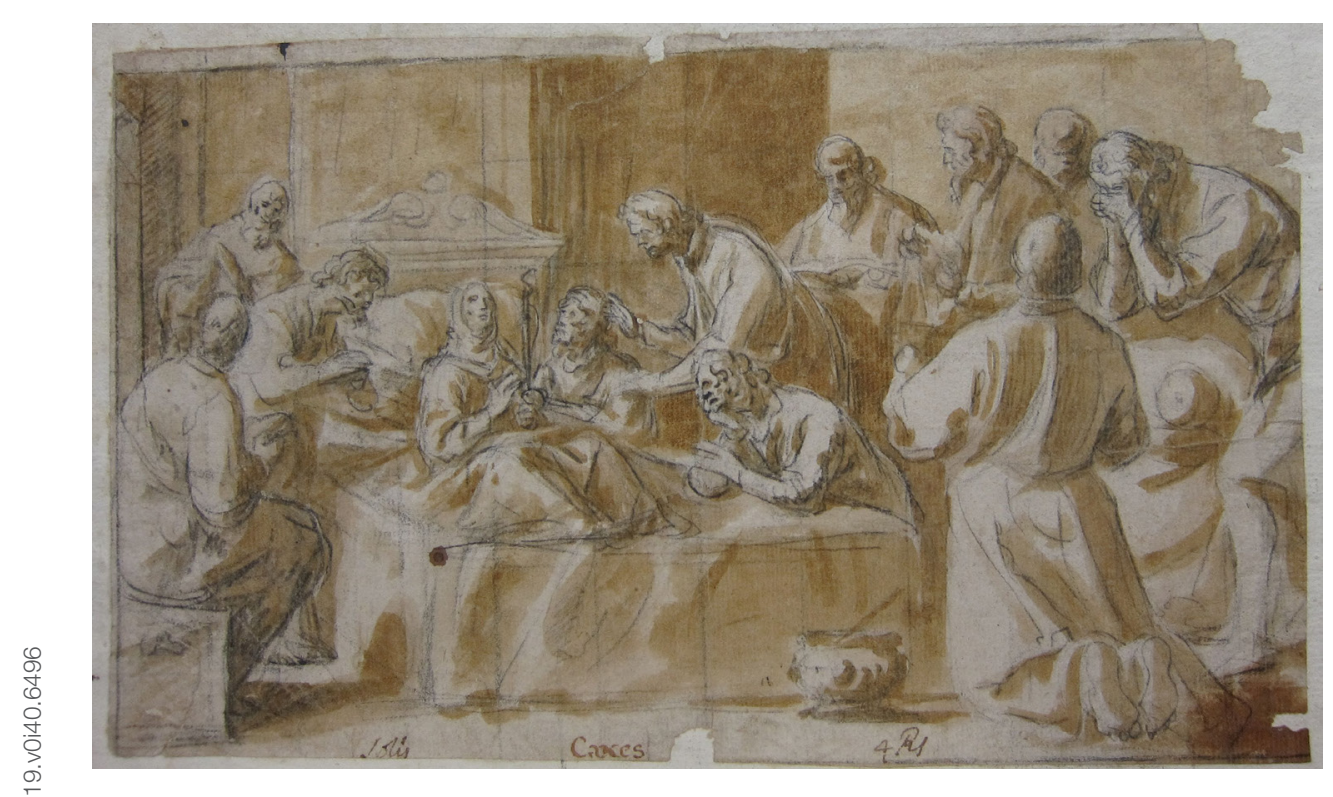

8. Eugenio Cajés,

Dormición de la

Virgen, colección

Colomer, Madrid

9. Eugenio Cajés,

Evangelista o Apóstol,

colección Félix Palacios,

Zaragoza

de Cajés en el empleo del lápiz negro, la sanguina y las abreviaciones físicas tan características del artista (Rodríguez, en Spanish Master Drawings, 2019: 14-17; Rodríguez, en El dibujo español, 2019: 50-53). Sin duda está directamente vinculado con el san Juan Evangelista de la Biblioteca Nacional y el san Mateo de la Real Academia Española procedente de la colección Rodríguez Moñino-Brey, con los que además coincide prácticamente en medidas, por lo que es probable que formasen parte de un mismo grupo de dibujos preparatorios para una serie de pinturas de los evangelistas de la que no tenemos constancia. Junto a ellos, hemos de mencionar la cercanía estilística y técnica con el Apóstol de la Biblioteca Nacional y el Apóstol caminando en el verso del Apóstol Santiago del Museo del Prado (Angulo y Pérez, 1977: 21, n. 58, 64, 65; 26, n. 100; Blas, Ciruelos y Matilla, 2002: 34-35, n. 4) ${ }^{24}$.

Para concluir, mencionaremos también un San Jerónimo, lienzo inédito firmado y fechado por Cajés en colección particular ${ }^{25}$. Aunque no hay dibujo preparatorio conocido, tal vez podría identificarse con el cuadro de esta misma iconografía que estuvo en El Escorial (Angulo y Pérez, 1969: 248, n. 175) [10]. Se relaciona asimismo con el lienzo de Barto- 
lomé Carducho pintado para Castrojeriz, así como con el dibujo de Vicente en el Courtauld Institute (Pascual y Rodríguez, 2015: 64-67, n. 4-5).

Vicente Carducho y Eugenio Cajés fueron dos figuras capitales en el ámbito artístico cortesano durante el primer tercio del siglo del siglo XVII, cuya labor de magisterio es clave en el desarrollo y evolución posterior de la pintura madrileña. En sus respectivos obradores se formaron algunos de los principales artistas de la generación siguiente, entre los que se encuentran Félix Castello ${ }^{26}$ y Jusepe Leonardo que, partiendo de sus enseñanzas, perpetuarán los métodos y procesos de raigambre italiana, incluyendo los trabajos al fresco. De todos ellos destaca sobre todo Francisco Rizi, hijo de Antonio Ricci (otro de los colaboradores de Zuccaro) y principal discípulo de Carducho, que prolongará su estela hasta finales de siglo, y que se convertirá en uno de los divulgadores del nuevo lenguaje grandilocuente del barroco deslumbrante y efectista que se desarrolla en el panorama artístico madrileño a partir de la segunda mitad del siglo XVII.

El relato de Palomino recogido en su biografía de Claudio Coello resulta muy revelador tanto de la importancia y la práctica habitual y constante del dibujo entre los artistas madrileños del Siglo de Oro, como del elevado valor que se le concedía dentro de los obradores de la época:

Hallabale muchas vezes su Maestro [Rizi] dibujando a horas desusadas [...] Tenía costumbre su Maestro de hazer en qualquiera papelillo algún rasguño, o apuntamiento de lo que se le ofrecia, ya fuesse de Historia, o ya fuesse de perspectiva, y luego los rompia, y los arrojaba, pero Claudio tenia gran cuidado de recogerlos, y juntarlos, y estudiar en ellos (Palomino, 1724: 440).

Algo parecido comenta de Isidoro Arrendondo, también discípulo de Rizi:

Murio Rici y dexole a Don Isidoro por heredero [...] estudio de Pintura, que era muy quantioso; pues solo de borroncillos, dibujos y trazas de Rizi no tenían numero, ni precio. Mucho le importó á Isidoro esta herencia, pues ayudado de este caudal, no se le ofrecía Obra en que no encontrase diseño,

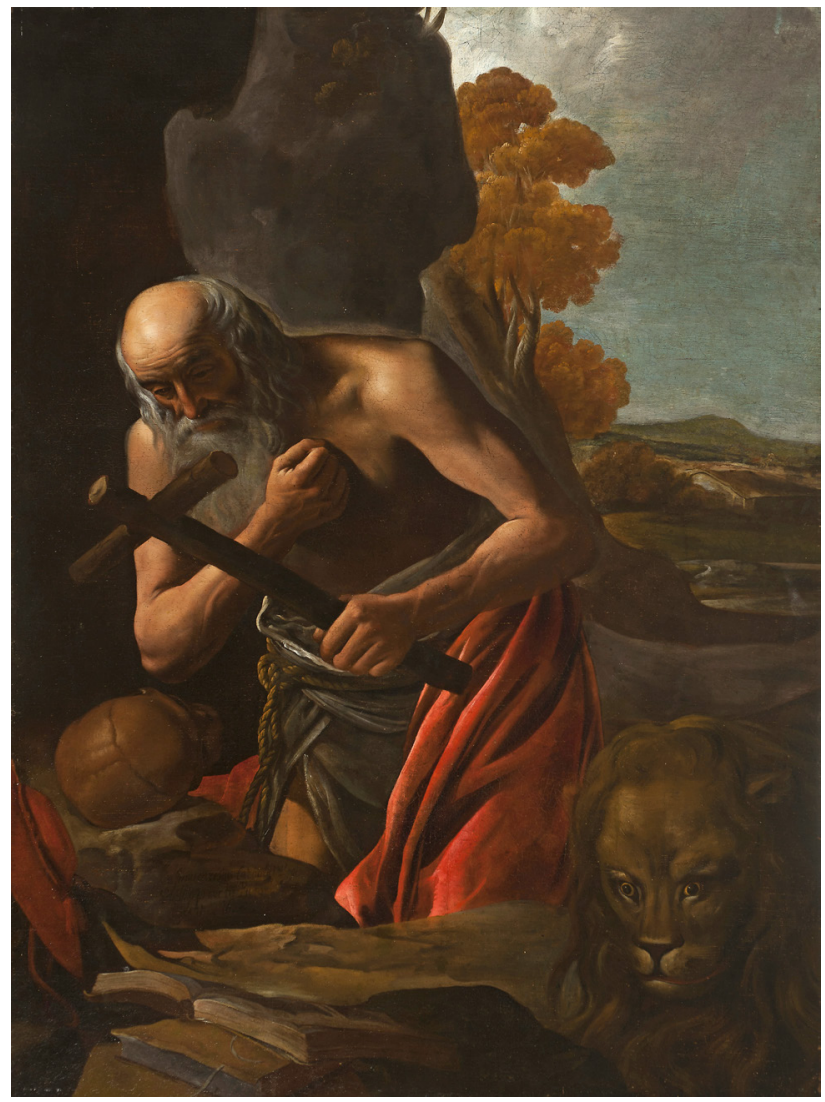

10. Eugenio Cajés, San Jerónino, colección particular

traza u dibujo de su maestro para su desempeño (Palomino, 1724: 461).

No parece resultar casual que ambos tuviesen como maestro a Francisco Rizi.

Estos testimonios vienen a ser prueba de que a finales del siglo XVII seguía vigente y poniéndose en práctica aquel principio de raíz italiana de que el dibujo es la base intelectual y científica de la instrucción y la creación artística. Carducho lo había transmitido a sus discípulos que habían hecho lo propio con los suyos, de tal modo que, como propugnaba desde el principio en sus Diálogos, cuando el discípulo preguntaba «Que haré (señor) para ser buen pintor?», el maestro le contestaba siempre: «Dibujar, especular, y más dibujar» (Carducho, 1633: fol. 2r.). 


\section{Notas}

1 Este artículo forma parte del proyecto de investigación Dibujos españoles de la Biblioteca Nacional de España. Escuela madrileña. Segunda mitad del siglo XVII (BNE-Fundación Universitaria Española), dirigido por Isabel Clara García-Toaño, Álvaro Pascual Chenel y Ángel Rodríguez Rebollo. Agradezco profundamente a las instituciones y colecciones que han cedido generosamente las fotografías que ilustran el presente estudio.

2 Se plantea para Cajés hacia 1595, aunque por el momento no hay constancia documental.

3 Óleo sobre lienzo, $209 \times 148 \mathrm{~cm}$.

4 Real Academia de Bellas Artes de San Fernando (RABASF). En: <http://xurl.es/7v6db> (fecha de consulta: 10-2-2019).

5 Con el fin de evitar repeticiones de notas, para todo lo relacionado con Carducho dibujante remitimos de modo general a Pascual y Rodríguez (2015). Para los dibujos conservados en los Uffizi véase además / Segni nel tempo (2016), pp. 158-189.

6 Gabinetto dei Disegni e delle Stampe, Galerie degli Ufiizi (GDSU). En: <http://xurl.es/uwttk> (fecha de consulta: 20-3-2019).

7 Staatliche Kunstsammlungen, Dresden (SKD). En: <bit.ly/2xfrBnL> (fecha de consulta: 6-6-2019).

8 Biblioteca Nacional de España (BNE. En: <http://xurl.es/p86ui> (fecha de consulta: 16-5-2019).

9 La bibliografía básica general sobre Cajés continúa siendo Angulo y Pérez, 1969: 212-259; Pérez, 1976: 307-316; Ángulo y Pérez, 1977: 13-27; Pérez, 1994: 1-10. Ahora también de modo más específico para sus dibujos, / Segni nel tempo, 2016: 137-151, n. 37-49.

10 Sobre la actividad de Eugenio Cajés véase además Pascual y Rodríguez, 2017: 2643-2730. Se anticipan aquí algunas de las novedades y resultados de un artículo más amplio sobre Eugenio Cajés que será publicado en breve.

11 Pascual y Rodríguez, 2015: 169-174, n. 29; Navarrete, 2016: 167-168, n. 64; Pascual en La colección Delgado, 2017: 60-63.

12 Esta práctica la encontramos en otras obras del artista, como en la figura esbozada a la izquierda en el dibujo de San Francisco de la BNE (DIB/15/1/15); o en el lienzo de la Flagelación de la colección Delgado tal como ha revelado la radiografía.

13 Angulo y Pérez, 1969: 239, n. 136; Angulo y Pérez, 1977: 15, n. 19-20; Pérez, 1994: 3-5; Véliz, 2011 b: n. 11.

14 Museo Nacional del Prado (MNP). En: <http://xurl.es/f1xlf> (fecha de consulta: 14-4-2019).

15 MNP. En: <http://xurl.es/oydn3> (fecha de consulta: 14-4-2019).

16 Alcalá Subastas, 13 y 14 de diciembre de 2017, lot. 286.

17 «Eugenius Cagesius Catholici Regis Philippi tertii pictor fecit anno 1616».

18 Alcalá subastas, 25 y 26 de mayo de 2016, lot. 374. Angulo y Pérez Sánchez, 1969: 230-231, n. 28; Palomino 1724: 401; Ponz, 1793 : 291.

19 MNP. En: <http://xurl.es/quopu> (fecha de consulta: 14-4-2019).

20 Subastas Segre, 19 de septiembre de 2017, lot. 53.

$21165 \times 270 \mathrm{~mm}$.

22 Angulo y Pérez, 1977: 52, n. 282-285; Navarrete, 2016: 190-191, n. 83; Pascual y Rodríguez, 2017: 2716.

23 Junto a Isabel Clara García-Toraño y Ángel Rodríguez Rebollo, preparamos un trabajo precisamente sobre la labor coleccionista de Francisco de Solís.

24 Los dibujos mencionados pueden verse en los siguientes enlaces: BNE. En: <http://xurl.es/fw5h4> (fecha de consulta: 5-6-2019). Real Academia Española (RAE). En: <http://xurl.es/rwsef> (fecha de consulta: 5-6-2019). BNE. En: <http://xurl.es/8a68f> (fecha de consulta: 5-6-2019). MNP. En: <http://xurl.es/j4kjw> (fecha de consulta: 5-6-2019).

25 Óleo sobre lienzo, 124,5 x 92,5, cm. «Eugeniu Caxesius Catholici Regis/Philippi tertii Pictor/ fecit.1620».

26 Recordemos además que su abuelo, Giovanni Battista Castello, el Bergamasco, había trabajado al servicio de Felipe Il, y que su padre Fabricio era otro de los artistas italianos llegados a El Escorial.

\section{Bibliografía}

AGULLÓ Y COBO, Mercedes (2003), «Un curioso coleccionista del siglo XVII: Don Manuel de Porras», Archivo Español de Arte, n. ${ }^{303,}$ pp. 235-248.

ANGULO Î̃̃IGUEZ, Diego y PÉREZ SÁNCHEZ, Alfonso (1969), Pintura madrileña del primer tercio del siglo XVII, CSIC, Madrid.

-(1977), A Corpus of Spanish Drawings, vol II: Madrid School 1600 to 1650, Londres.

BANNER, Lisa (2007), «Francisco de Solís: A Seventeenth-Century Artist-Collector in Madrid», Master Drawings, vol. 45, n. ${ }^{\circ}$ 3, pp. $359-366$.

BLAS, Javier, CIRUELOS y ASCENSIÓN MATILLA, José Manuel (2002), Colección Rodríguez Moñino-Brey, Real Academia Española. Dibujos, Madrid.

CALVO RUATA, José Ignacio (dir.) (2019), El dibujo español en el gusto privado. Del Renacimiento a la llustración, catálogo de la exposición, Fundación Goya en Aragón, Zaragoza.

CARDUCHO, Vicente (1633), Diálogos de la Pintura. Su defensa, origen, esencia, definición, modos y diferencias..., Madrid. 
GARCÍA-TORAÑO, Isabel Clara, MEZTE, Gudula, PASCUAL CHENEL, Álvaro and RODRíGUEZ REBOLLO, Ángel (2019), «An Unpublished Drawing by Vicente Carducho for the Series at El Paular», Master Drawings, 57, 2, pp. 197-204.

GIMILIO, David y GÓMEZ FRECHINA, José (com.) (2017), La colección Delgado, catálogo de la exposición, Museo de Bellas Artes de Valencia, Valencia.

NAVARRETE PRIETO, Benito (dir.) (2016), I Segni nel tempo. Dibujos españoles de los Uffizi, catálogo de la exposición, Fundación Mapfre, Madrid.

PALOMINO, Antonio (1724), El Museo pictórico y escala óptica. Tomo IIl con las vidas de los pintores y estatuarios eminentes españoles, Madrid.

PASCUAL CHENEL, Álvaro (2017), «Un San Bartolomé poco conocido de Vicente Carducho en Londres», Archivo Español de Arte, n. 360 , pp. 429-436.

- (2018), «I Carducho e i Cajés», en FAIETTI, Marzia, GALLORI, Corinna e MOZZATI, Tommaso (a cura di) Spagna e Italia in dialogo nell’Europa del Cinquecento, catalogo della mostra, Galerie degli Uffizi, Giunti editore, Florencia, pp. 224-231.

PASCUAL CHENEL, Álvaro y RODRíGUEZ REBOLLO, Ángel (2015), Vicente Carducho. Dibujos. Catálogo razonado, Centro de Estudios Europa Hispánica, Madrid.

- (2017) «¿A la sombra de Velázquez? Los pintores cortesanos durante el reinado de Felipe IV», en MARTíNEZ MILLÁN, José y RIVERO RODRíGUEZ, Manuel (dirs.), La Corte de Felipe IV (1621-1665). Reconfiguración de la Monarquía Católica. Arte, coleccionismo y sitios reales, Polifemo, Madrid, tomo III, vol. 4, pp. 2643-2730.

PÉREZ SÁNCHEZ, Alfonso (1969/2003), Catálogo de la colección de Dibujos del Instituto Jovellanos de Gijón, Madrid.

- (1976), «Pintura madrileña del siglo XVI: Addenda», Archivo Español de Arte, n. ${ }^{0}$ 195, pp. 293-326.

- (1986), Historia del dibujo en España de la Edad Media a Goya, Cátedra, Madrid.

- (1994), «Eugenio Cajés, adenda et corrigenda», Archivo Español de Arte, n. ${ }^{\circ}$ 265, pp. 1-10.

PONZ, Antonio (1793), Viage de España, tomo V, Madrid.

RAMÓN, Artur y RODRíGUEZ REBOLLO, Ángel (dirs.) (2019), Spanish Master Drawings: From Cano to Picasso, exh. Cat, Artur Ramon Art, Barcelona. 